\title{
RELATIONS IN STABLE HOMOTOPY MODULES
}

\author{
DONALD W. KAHN ${ }^{1}$
}

\begin{abstract}
The "generating hypothesis" of Freyd implies various fascinating statements in stable homotopy, particularly about the stable homotopy ring of spheres and the modules over this ring. The present paper extends some of these consequences. It also investigates the question of whether a possible counterexample to the hypothesis might be detected by its action on homology groups; for this, we find a connection with work of Joel Cohen on coherent graded rings.
\end{abstract}

In his basic paper [2], P. Freyd has put forth a conjecture, known as the "generating hypothesis", which says that if a map $f: X \rightarrow Y$, between finite, connected complexes $X$ and $Y$, induces the zero map on stable homotopy groups, then $f$ is stably null-homotopic. The conjecture, unresolved at the time of this writing, is remarkable, because of the wealth of statements in stable homotopy theory which are known to follow from it. Some of these statements have been verified in many cases (see, for example [4]), but many of them, while of great interest, remain a complete mystery. They may be equivalent to the conjecture itself; in fact, various equivalent statements are now known [5].

One of these statements, which is a consequence of the "generating hypothesis", asserts that if $\alpha$ and $\beta$ are elements of $p$-torsion in the stable homotopy ring of spheres, there is a nonzero $\gamma$ which is simultaneously divisible by $\alpha$ and $\beta$ (see Proposition 9.3 in [2]). The purpose of the present paper is to study extensions and generalizations of this statement and to prove related theorems which follow without invoking the presently unproved "generating hypothesis". We first prove a mild generalization of this particular statement for the stable homotopy ring $G_{*}=\pi_{*}^{S}\left(S^{0}\right)$, and then investigate conditions which assure a similar statement for the stable homotopy of a finite complex $X$. Next, we prove that either there are uncountably many counterexamples to one of the consequences of the "generating hypothesis", or at most finitely-many elements are unrelated to other independent elements via the existence of nonzero common

Received by the editors November 13, 1972 .

AMS (MOS) subject classifications (1970). Primary 55E10.

Key words and phrases. Stable homotopy modules, generating hypothesis.

${ }^{1}$ Work partially supported by centract NSF GP 34021 .

(c) American Mathematical Society 1973 
multiples. This result is largely algebraic here, but relates to earlier work on infinite generation [4]. Our final theorem, independent of the "generating hypothesis", considers the question of when a map between finite complexes, which induces a nonzero homomorphism on homology, need induce a nonzero homomorphism on stable homotopy groups. We are able to show that either this is the case, or else certain density conditions hold in the stable homotopy of the domain space. This result, which uses the techniques of [1], is aimed at the question of searching for suitable invariants to detect a possible counterexample to the "generating hypothesis".

While from the algebraist's viewpoint, the results of this paper will appear weak, one should remember that our knowledge of the stable homotopy ring is still scanty; thus, results on modules over the ring $G_{*}$ will necessarily present serious difficulties.

We begin with our generalization of Freyd's result.

THEOREM 1. Let the elements $\alpha_{1}, \cdots, \alpha_{n}, \beta_{1}, \cdots, \beta_{m}$ belong to the ring $G_{*}=\pi_{*}^{S}\left(S^{0}\right)$, all being nonzero, p-torsion elements for some fixed prime $p$. Assume the "generating hypothesis". Then there is a nonzero element $x \in G_{*}$ such that

(1) $x=\alpha_{i} \cdot \gamma_{i}, \gamma_{i} \in G_{*}, 1 \leqq i \leqq n$,

(2) $x \cdot \beta_{j}=0,1 \leqq j \leqq m$,

(3) $p \cdot x=0$.

Proof. Choose $M$ much larger than the degree of each element in question, and form a finite complex $Y$ as follows:

$$
Y=\left(S^{M} \cup e^{a_{1}}\right) \vee \cdots \vee\left(S^{M} \cup e^{a_{n}}\right) \vee S^{b_{1}} \vee \cdots \vee S^{b_{m}} \vee S^{M I},
$$

where $a_{i}=\operatorname{deg}\left(\alpha_{i}\right)+M+1, b_{j}=M-\operatorname{deg}\left(\beta_{j}\right)$, and the attaching maps for the first $n$ summands are the elements $\alpha_{i}$, or rather their representatives. Take a map $f: S^{M} \rightarrow Y$ to be the inclusion of $S^{M}$ in the first $n$ summands, $\beta_{i}$ on the next $m$ summands, and $p \cdot 1_{S^{M}}$ on the last summand. We note that an element $x \in \pi_{*}^{S}\left(S^{M}\right)$ is in the kernel of the induced homomorphism $\pi_{*}^{S}\left(S^{M}\right) \rightarrow \pi_{*}^{S}\left(S^{M} \cup e^{a_{i}}\right)$ precisely when it is divisible by $\alpha_{i}$. Hence, $x \in \pi_{*}^{S}\left(S^{M}\right)-\{0\}$ will satisfy conditions (1), (2), and (3) if and only if $f_{\#}^{S}(x)=0$.

Suppose for a contradiction that $f_{\#}^{S}$ is a monomorphism on stable homotopy groups. Then in the Puppe sequence

$$
S^{M} \stackrel{f}{\longrightarrow} Y \stackrel{p}{\longrightarrow} C f \stackrel{d}{\longrightarrow} S^{M+1} \longrightarrow \cdots
$$

we have that $d_{\#}^{S}$ is the zero map. By the "generating hypothesis", $d$ is stably null-homotopic. Using the corresponding Puppe sequence of stable 
mapping groups, mapping from $C f$, we see that $p$ has a stable right inverse. Thus,

as $G_{*}$-modules.

$$
\pi_{*}^{S}(Y)=\pi_{*}^{S}\left(S^{M I}\right) \oplus \pi_{*}^{S}(C f)
$$

We shall complete the proof by showing that no $G_{*}$-module homomorphism $\pi_{*}^{S}(Y) \rightarrow \pi_{*}^{S}\left(S^{M}\right)$ can be a left inverse for $f_{\# \text {. }}^{S}$

In the wedge decomposition for $Y$, the first $n$ summands have the form $S^{M} \cup e^{a_{i}}$. Because the attaching maps are $p$-torsion, any conjectured splitting, restricted to such a summand, would induce a multiple of a power of $p$ on the homotopy group in dimension $M$. The summands $S^{b_{j}}$ must be sent to zero by a splitting, for reasons of dimension. We suppose that on the group $\pi_{M}^{S}$, restricted to the last summand, the splitting acts as multiplication by some integer $c$.

Writing the conjectured splitting as $g$, we may calculate $g \cdot f_{\#}^{S}\left(1_{S} S^{M}\right)=$ $g\left(i_{1}, \cdots, i_{n}, 0, \cdots, 0,0\right)+g\left(0, \cdots, 0, p \cdot 1_{S^{M}}\right)$. Here, we have used $i_{j} \cdot S^{M} \rightarrow S^{M} \cup e^{a_{j}}$ for the inclusion. By our remarks above, the first term is divisible by $p$, while the last is precisely $p \cdot c$. Thus, $g \cdot f_{\#}^{S}$ is divisible by $p$, showing that no splitting could occur, and completing the proof.

Now, we wish to investigate when two elements in a stable homotopy module, say $x, y \in \pi_{*}^{S}(X), X$ being a finite, connected complex, have a nonzero common multiple. If we look at examples of the form $X=Y \vee Z$, it is clear that some conditions will be necessary to insure that this is the case. The most straightforward generalization of Proposition 9.3 in [2] would be

Proposition 1. Suppose that the "generating hypothesis" is true. Let $X$ be a connected finite complex, with $\alpha_{1}, \alpha_{2}$ homogeneous elements in the p-torsion of $\pi_{*}^{S}(X)$. Suppose that there is an $M$ so that any map $f: X \cup_{\alpha_{i}} e^{a_{i}} \rightarrow X, a_{i}=\operatorname{dim} \alpha_{i}+1, i=1,2$, acts on $\pi_{M}^{S}$ as multiplication by $a$ multiple of a power of $p$. Then, $\alpha_{1}$ and $\alpha_{2}$ have a nonzero common multiple.

The proof is similar to the above.

We wish, however, to investigate relations in stable homotopy modules, without invoking the "generating hypothesis". To this end, we make the following definition; this works in any graded module, but we are principally interested in the case of modules $\pi_{*}^{S}(X)$ over the ring $G_{*}=\pi_{*}^{S}\left(S^{0}\right)$.

Definition 1. Let $\alpha \in \pi_{*}^{S}(X)$ generate an additive summand. Let $(\alpha)$ denote the submodule generated by $\alpha$. We say that $\alpha$ is an unrelated element, if $(\alpha)$ is a module direct summand of $\pi_{*}^{S}(X)$.

A generator of $\pi_{*}^{S}\left(S^{n_{1}} \vee \cdots \vee S^{n_{k}}\right)$, corresponding to one of the spheres, is an unrelated element. Proposition 2 and the remarks which follow it below show that if the "generating hypothesis" is true, there are probably no other examples. Our goal, however, is to study the situation, without 
invoking the "generating hypothesis". Nevertheless, we suspect that unrelated elements should in some sense be rare.

LemMA 1. Let $\alpha_{1}, \alpha_{2}, \cdots, \alpha_{k}$ be a family of distinct, unrelated elements. Then they generate a module direct summand of $\pi_{*}^{S}(X)$ which is isomorphic to $\sum \oplus G_{*} / \operatorname{ker}\left(i_{n}\right)$ where $i_{n}: G_{*} \rightarrow \pi_{*}^{S}(X)$ is given by $i_{n}(1)=\alpha_{n}$.

The proof is obvious.

THEOREM 2. Let $X$ be a finite, connected complex. Then either

(a) There are at most finitely-many unrelated elements in $\pi_{*}^{S}(X)$, or

(b) there are uncountably many $G_{*}$ homomorphisms $\pi_{*}^{S}(X) \rightarrow \pi_{*}^{S}(X)$, which are not induced by geometric maps.

Proof. If we assume that (a) is false, then there are infinitely many, nonzero summands of $\pi_{*}^{S}(X)$. Observe that we can construct uncountably many $G_{*}$ homomorphisms $\pi_{*}^{S}(X) \rightarrow \pi_{*}^{S}(X)$ by choosing either the identity map or the zero map on each summand. It will then suffice to show that there are only countably many stable homotopy classes of maps from $X$ to itself. This is easily done by induction, using either Postnikov systems or the cellular structure of $X$.

REMARKS. The negation of the first assertion implies, of course, that $\pi_{*}^{S}(X)$ is infinitely generated. This is known, however, to be true in many cases (see [4]). On the other hand, statement (b) contradicts a consequence of the "generating hypothesis", which asserts that the functor $\pi_{*}^{S}(-)$ is a full imbedding. I would conjecture that $\pi_{*}^{S}(X)$ can have a nontrivial module summand, only when $X$ decomposes as a wedge of spaces. The following proposition throws some light on this question.

Proposition 2. Suppose we have a module decomposition $\pi_{*}^{S}(X)=$ $M \oplus N$, where $M$ is the stable homotopy module of some finite, connected complex $Y$. If the "generating hypothesis" is true, then $X$ has the stable homotopy-type of $Y \vee Z$, where $M=\pi_{*}^{S}(Y)$ and $N=\pi_{*}^{S}(Z)$.

Proof. Because $\pi_{*}^{S}(-)$ is a full-imbedding, the inclusion and projection for the summand $M$ of $\pi_{*}^{S}(X)$ are represented by geometric maps. Regarding $Y$ as a subcomplex of $X$, one easily sees from the five-lemma that $\pi_{*}^{S}(X / Y)=N$.

Clearly, most modules with 1 generator cannot be the module of a finite complex. As a final remark, we note that the "generating hypothesis" implies that the image of a monomorphism, $\pi_{*}^{S}(Y) \rightarrow \pi_{*}^{S}(X)$, which is necessarily then induced by a geometric map, must be a module summand of $\pi_{*}^{S}(X)$.

We wish finally to consider the question of how one might try to detect possible counterexamples to the "generating hypothesis". There is some 
information at present (see [5]) that implies that such examples, if they exist at all, will be elusive. On the other hand, one would reinforce the "generating hypothesis" if one could show that a map which induces zero on stable homotopy groups must also induce zero on some other invariants, such as homology. Or equivalently, it would be pleasant to know that if $f$ induced a nonzero map on homology groups, then for some dimension, it induced a nonzero map on stable homotopy groups. Although we cannot quite do this, our last theorem, which is motivated by, although independent of, the "generating hypothesis", offers a step in this direction.

The work of Joel Cohen [1] shows that in a space with finitely many nonvanishing homotopy groups, whenever we are given an element in cohomology with $Z_{p}$ coefficients, infinitely many Steenrod operations are nonzero on this element. By considering the lowest dimensional nonzero such operation, in a dimension bigger than that of the complex which we are studying, we formulate a sort of minimum possible density for stable homotopy groups for our complex (see case (a) of the following theorem). Our theorem then says that if we are given a map $f: X \rightarrow Y$ which is nonzero on homology with coefficients in $Z_{p}, p$ a prime, then either $X$ always has more dense stable homotopy than would follow from Cohen's result applied to classes in the image of $f^{*}$, or the map on stable homotopy groups, tensored with $Z_{p}$, is nonzero.

We shall speak of stable Postnikov systems. One can either view them as ordinary Postnikov systems of some suitable suspension of our spaces, or in terms of spectra. For this latter point of view, see [1].

THEOREM 3. Let $f: X \rightarrow Y$ be a map between finite, connected complexes. Let $p$ be prime, and we suppose that there is $n>0$ so that $f^{*}: H^{n}\left(Y ; Z_{p}\right) \rightarrow$ $H^{n}\left(X ; Z_{p}\right)$ is nonzero.

Let $X_{i}$ refer to a stable ith Postnikov term for $X$. Choose $u \in H^{n}\left(Y ; Z_{p}\right)$ so that $f^{*}(u)=v \neq 0$. With $i>\max (\operatorname{dim} X, \operatorname{dim} Y)$, take representing classes $\bar{u}, \bar{v}$ for $u, v$ in the cohomology of the ith Postnikov terms, so that $f_{j}^{*}(\bar{u})=\bar{v} \neq 0$. Using [1], choose a Steenrod operation $\mathscr{P}$ of lowest degree such that $\mathscr{P}(\bar{v}) \neq 0$, and $\operatorname{dim}(\mathscr{P}(\bar{v}))>i$. We write $\operatorname{dim}(\mathscr{P}(\bar{v}))=n(i)$.

Then at least one of the following holds.

(a) For every $i>\max (\operatorname{dim} X, \operatorname{dim} Y)$, there is a $j, i<j<n(i)-1$, with $\pi_{j}^{S}(X) \otimes Z_{p} \neq 0$.

(b) $f_{\#}^{S}: \pi_{*}^{S}(X) \otimes Z_{p} \rightarrow \pi_{\#}^{S}(Y) \otimes Z_{p}$ is nonzero.

Proof. Assume that (a) is false, and that $i$ designates the first such case. I claim that $\mathscr{P}(\bar{u})$ lifts to a nonzero element in the cohomology of $Y_{n(i)-2}$. For if not, it is in the image of the transgression of some Postnikov fibration, with fibre $K\left(\pi_{j}^{S}(Y), j\right), j<n(i)-1$. Applying the appropriate 
induced $\operatorname{map} f_{j}^{*}$ (see [3]), we see that $\mathscr{P}(\bar{v})$ is annihilated when projected up to the cohomology of $X_{n(i)-2}$. This is not compatible with our assumption that (a) is false.

Now, for dimension reasons, $\mathscr{P}(\bar{u})$ cannot survive to the cohomology of $Y_{n(i)-1}$, for else it will remain in $H^{*}\left(Y ; Z_{p}\right)$. Hence, there is $\iota \in$ $H^{n(i)-1}\left(\pi_{n(i)-1}^{S}(Y), n(i)-1 ; Z_{p}\right)$ such that $\tau(\iota)=\mathscr{P}\left(\bar{u}^{\prime}\right)$, where $\bar{u}^{\prime}$ is the image of $\bar{u}$ in $H^{n(i)}\left(Y_{n(i)-2} ; Z_{p}\right)$.

We consider now the following map of fibre spaces:

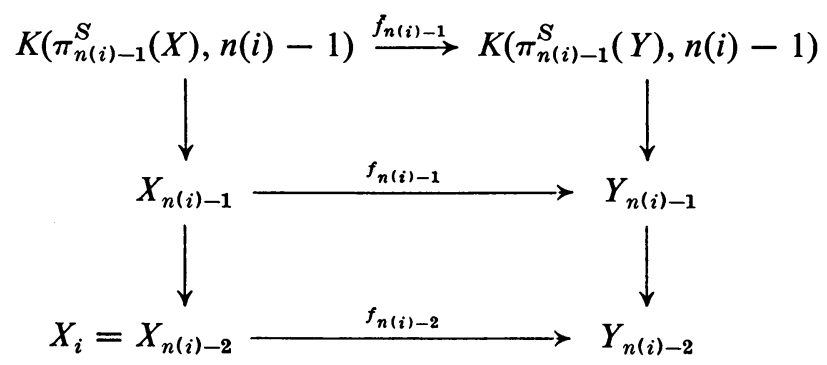

Hence, we have $\tau\left(\bar{f}_{n(i)-1}^{*}(\iota)\right)=f_{n(i)-2}^{*} \tau(i)=f_{n(i)-2}^{*}\left(\mathscr{P}\left(\bar{u}^{\prime}\right)\right)$. From the diagram

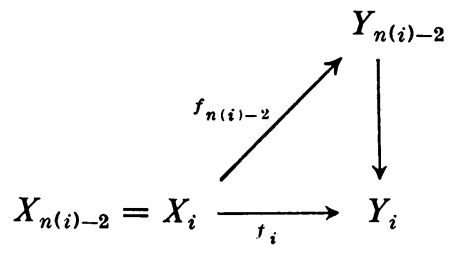

we get $\tau\left(\bar{f}_{n(i)-1}^{*}(\iota)\right)=f_{i}^{*}(\mathscr{P}(\bar{u}))$.

But, from our earlier remarks, we know that the right hand side is nonzero. If $f_{\sharp}^{S} \otimes 1$ were zero, using the Hurewicz isomorphism and taking the dual vector space, we get that the left hand side is zero. Thus, $f$ must induce a nonzero homomorphism on stable homotopy groups, tensored with $Z_{p}$, completing the proof.

REMARKS. (1) We note here that it follows easily from the results of [3] that if $k_{y}^{i+2}$ denotes the stable $k$-invariant, and $f_{\#}^{S}=0$, then $f_{i}^{*}\left(k_{y}^{i+2}\right)=0$.

(2) In the above theorem, one would like to prove (b) directly. Other new methods will clearly be necessary for this. One disadvantage of our method is that it is tied down to a specific $u$ so that $f^{*}(u) \neq 0$. Hopefully, more delicate methods can be found to get around these problems.

\section{BIBLIOGRAPHY}

1. Joel Cohen, Coherent graded rings and the non-existence of spaces of finite stable homotopy-type, Comment, Math. Helv. 44 (1969), 217-228. MR 40 \#892. 
2. P. Freyd, Stable homotopy, Proc. Conference on Categorical Algebra (La Jolla, Calif., 1965), Springer-Verlag, New York, 1966, pp. 121-172. MR 35 \#2280.

3. Donald W. Kahn, Induced maps for Postnikov systems, Trans. Amer. Math. Soc. 107 (1963), 432-450. MR 27 \#764.

4. - A note on stable homotopy modules, Proc. Amer. Math. Soc. 26 (1970), 683-686. MR 42 \#5257.

5. - Stable spectral sequences and their applications, Amer. J. Math. (2) 94 (1972), 1131-1154.

School of Mathematics, University of Minnesota, Minneapolis, Minnesota 55455 